\title{
Algebraic Methods for Direct and Feature Based Registration of Diffusion Tensor Images
}

\author{
Alvina Goh and René Vidal \\ Center for Imaging Science, Department of BME, Johns Hopkins University, \\ 308B Clark Hall, 3400 N. Charles St., Baltimore, MD 21218, USA \\ agoh@jhu.edu, rvidal@cis.jhu.edu
}

\begin{abstract}
We present an algebraic solution to both direct and featurebased registration of diffusion tensor images under various local deformation models. In the direct case, we show how to linearly recover a local deformation from the partial derivatives of the tensor using the so-called Diffusion Tensor Constancy Constraint, a generalization of the brightness constancy constraint to diffusion tensor data. In the feature-based case, we show that the tensor reorientation map can be found in closed form by exploiting the spectral properties of the rotation group. Given this map, solving for an affine deformation becomes a linear problem. We test our approach on synthetic, brain and heart diffusion tensor images.
\end{abstract}

\section{Introduction}

Diffusion Tensor Imaging (DTI) is a relatively new 3-D imaging technique that measures the diffusion of water molecules in human and animal tissues. As the directional dependence of water diffusion rates is closely related to the structural anisotropy of the medium, DTI can be potentially used to infer the organization and orientation of tissue components. This has generated much enthusiasm and high expectations, because DTI is presently the only available approach to noninvasively study the three-dimensional architecture of white matter tracts, and quantify physical and geometrical properties of neuronal fibers in vivo.

Unfortunately, current image processing and computer vision algorithms are unable to take full advantage of what DTI offers. The main reason is that, unlike conventional images, DTI not only measures the intensity at each voxel, but also the orientation. Orientation at each voxel is represented mathematically with a symmetric positive semi-definite (SPSD) tensor field $D: \mathbb{R}^{3} \rightarrow \operatorname{SPSD}(3) \subset \mathbb{R}^{3 \times 3}$ that measures the diffusion in a direction $\boldsymbol{v} \in \mathbb{R}^{3}$ as $\boldsymbol{v}^{T} D \boldsymbol{v}$. Since the image data live on a 6 -dimensional space with nontrivial geometry, problems such as filtering, smoothing, edge detection, matching, segmentation, registration, etc., need to be reconsidered in light of the new mathematical structure of the data.

Up until now, most of the research on DTI has focused on fiber tracking and segmentation. Fiber tracking refers to the problem of extracting 3-D curves on the image that follow the main orientation of the tensor field at each voxel. By assuming that the largest principal axis of the diffusion tensor (DT) aligns with the predominant fiber orientation, one can obtain a 3 -D vector field representing 
the fiber orientation at each voxel. Fiber tracking is then equivalent to finding integral curves of this vector field. Existing fiber tracking methods include streamline techniques [1, tensor deflection [2, PDE-based curve evolution [3, 4, and dynamic programming [5]. Segmentation refers to the problem of grouping the fibers into tracts. For example, in images of the spinal cord, bundles of fibers have different functions, and one would like to cluster all fibers having the same or similar functions. Existing segmentation methods either assume that fiber tracts have already been extracted and segment these curves according to a certain cost function, or else segment the tensor data directly using various metrics on $\operatorname{SPSD}(3)$ 6, 7], such as the Euclidean distance between two fibers, or the ratio of the length of corresponding portions of the fibers to the overall length of the pairs 8]. In 9, fibers are reduced to a feature vector extracted from the statistical moments of the fibers, and segmentation is done by applying normalized cuts [10] to these feature vectors. [11] first reduces tensor data to a scalar anisotropic measure, and then applies a level set segmentation method.

Although registration of conventional 2-D and 3-D scalar images is a relatively well understood problem, registration of DT images is a problem that has received much less attention. The main difference between registration of scalar images and registration of tensor images is that in addition to estimating a local deformation model, e.g., translational, rigid or affine, one must also reorient each tensor so that it remains consistent with the surrounding anatomical structure in the image. In 12, several tensor reorientation approaches are proposed. The most commonly used method is the Finite Strain scheme [7, 13, 14], which, given an affine transformation $A$, reorients the tensor using the rotational component of $A$. Existing methods for registration of DT images are based on minimizing a cost function [15], such as sum-of-squared differences [13, 14, correlation [13], Euclidean distance [7] or diffusion profile [7, under an affine deformation model combined with the finite strain reorientation method. However, such methods are usually computationally intensive, and require good initialization.

The objective of this paper is to develop simple linear registration algorithms that can be used for initializing computationally intensive methods. The main contribution is to show that for the standard Euclidean metric in $\operatorname{SPSD}(3)$, the DTI registration problem can be solved in closed form, both directly from diffusion tensor data as well as from feature-point correspondences. Our direct approach is based on the so-called Diffusion Tensor Constancy Constraint (DTCC), a generalization of the well-known brightness constancy constraint (BCC) to DT data. We show that for various local deformation models, such as translational, rigid, and affine, together with the finite strain reorientation scheme, the DTCC leads to a linear relationship between the parameters of the deformation, the DT data and its first order partial derivatives. Our feature-based approach assumes that we are given a set of point correspondences in two images. We show that the tensor reorientation map can be computed directly from the singular value decompositions (SVD) of two corresponding tensors. Once this map has been computed, solving for a rigid or affine deformation becomes a linear problem. We test our approach on synthetic, brain and heart DT images. 


\section{Direct Registration of Diffusion Tensor Images}

In this section, we present an algebraic method for registering two diffusion tensor images $D_{1} \in \operatorname{SPSD}(3)$ and $D_{2} \in \operatorname{SPSD}(3)$ under various local deformation models. We also extend our method to a multi-resolution framework using a coarse-to-fine refinement strategy.

We assume that the coordinates of the voxels in the two images, $\boldsymbol{x}_{1}, \boldsymbol{x}_{2} \in \mathbb{R}^{3}$, are locally related by an affine deformation model

$$
\boldsymbol{x}_{2}=A \boldsymbol{x}_{1}+\boldsymbol{t}
$$

where $A \in \mathbb{R}^{3 \times 3}$ and $\boldsymbol{t} \in \mathbb{R}^{3}$. This local deformation model not only transforms the voxel coordinates, but also reorients the tensor data. We model the tensor reorientation with the Finite Strain (FS) method [7, 13, 14, which uses the Polar Decomposition Theorem [16] to express the affine matrix $A$ as the product of a rotation matrix $R \in S O(3)$ and a strain matrix $S \in \operatorname{SPSD}(3)$, i.e. $A=R S$. The tensor is then reoriented using the rotational component of $A$ as

$$
D_{2}=R D_{1} R^{\top}
$$

By combining the local deformation model with the tensor reorientation model, we obtain the following Diffusion Tensor Constancy Constraint (DTCC)

$$
D_{2}(A \boldsymbol{x}+\boldsymbol{t})=R D_{1}(\boldsymbol{x}) R^{\top} .
$$

In order to locally register $D_{1}$ and $D_{2}$ in the presence of noise, at each voxel $\boldsymbol{y}$ we seek the parameters $(A, \boldsymbol{t})$ that minimize the Frobenius norm of the error between the two tensors

$$
J=\sum_{\boldsymbol{x} \in \Omega(\boldsymbol{y})} \operatorname{trace}\left(D_{2}(A \boldsymbol{x}+\boldsymbol{t})-R D_{1}(\boldsymbol{x}) R^{\top}\right)^{2},
$$

where $\Omega(\boldsymbol{y}) \subset \mathbb{R}^{3 \times 3}$ is a neighborhood of voxel $\boldsymbol{y}$ at which the affine model is valid. While there are many possible metrics in $\operatorname{SPSD}(3)$ 6, 7, we have chosen the Frobenius norm, because it enables us to solve the registration problem in closed form for various 3-D deformation models, such as translational, rigid and affine, as we will show in the next subsections.

\subsection{3-D Translational Model}

In this subsection, we assume that the deformation is translational, i.e. $A=R=$ $S=I$. Under this deformation model, the DTCC (3) reduces to

$$
D_{2}(\boldsymbol{x}+\boldsymbol{t})=D_{1}(\boldsymbol{x}) .
$$

After expanding the left hand side in Taylor series, we obtain

$$
D_{2}(\boldsymbol{x}+\boldsymbol{t}) \approx D_{2}(\boldsymbol{x})+D_{x} d_{1}+D_{y} d_{2}+D_{z} d_{3},
$$


where $\boldsymbol{x}=(x, y, z)^{\top}, \boldsymbol{t}=\left(d_{1}, d_{2}, d_{3}\right)^{\top}$, and $\left(D_{x}, D_{y}, D_{z}\right)$ are the partial derivatives of the diffusion tensor at $\boldsymbol{x}$. Substituting (6) in (5) yields the following differential DTCC

$$
D_{x} d_{1}+D_{y} d_{2}+D_{z} d_{3}+D_{t}=\mathbf{0}_{3 \times 3} .
$$

Notice that (7) is a natural generalization of the well-known brightness constancy constraint (BCC) $I_{x} u+I_{y} v+I_{t}=0$ from scalar 2-D images $I(x, y, t)$ to DT images $D(x, y, z, t)$. However, an important difference is that while the BCC provides one equation in two unknowns, the DTCC provides 6 equations in 3 unknowns, because $D_{x}, D_{y}, D_{z}$ are $3 \times 3$ symmetric matrices.

Thanks to the DTCC, we may rewrite our cost function (4) as:

$$
J=\sum_{\Omega} \operatorname{trace}\left(D_{x} d_{1}+D_{y} d_{2}+D_{z} d_{3}+D_{t}\right)^{2} .
$$

After differentiating $J$ with respect to $d_{1}$, we obtain

$$
\frac{\partial J}{\partial d_{1}}=2 \sum_{\Omega} \operatorname{trace}\left(D_{x}\left(D_{x} d_{1}+D_{y} d_{2}+D_{z} d_{3}+D_{t}\right)\right)
$$

and similarly for $d_{2}$ and $d_{3}$. By setting these derivatives to zero, we can linearly solve for the displacement $\boldsymbol{u}=\left(d_{1}, d_{2}, d_{3}\right)^{\top}$ from

$$
G \boldsymbol{u}=-\boldsymbol{b},
$$

where

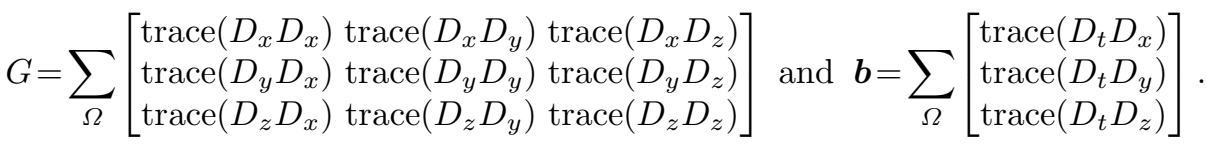

The similarity with the case of 2-D scalar images, where $\boldsymbol{u}$ is computed from a linear system of the form (9) with $G=\sum_{\Omega}\left[\begin{array}{cc}I_{x}^{2} & I_{x} I_{y} \\ I_{x} I_{y} & I_{y}^{2}\end{array}\right]$ and $\boldsymbol{b}=\sum_{\Omega}\left[\begin{array}{c}I_{t} I_{x} \\ I_{t} I_{y}\end{array}\right]$, is immediate. However, in the scalar case $\operatorname{rank}(G)=1$ when $\Omega$ consists of a single pixel, while in the tensor-valued case $G \in \mathbb{R}^{3 \times 3}$ is full rank for a generic tensor field $D$, even if $\Omega$ consists of a single voxel. Hence, with generic data one can solve for $\boldsymbol{t}$ at each voxel independently. Obviously, in the presence of noise estimating $\boldsymbol{t}$ from a single voxel is not robust, thus in practice one assumes that $\boldsymbol{t}$ is constant on a neighborhood $\Omega$ of size much larger than one.

\section{$2.2 \quad 3-D$ Rigid Model}

Assume now that the deformation model is a rigid-body transformation, i.e. $A=R \in S O(3)$ and $S=I$, and let $\boldsymbol{x}=(x, y, z)^{\top}$ and $\boldsymbol{t}=\left(d_{1}, d_{2}, d_{3}\right)^{\top}$. In this case, we use the well-known first order approximation [17] of the rotational component of the deformation $R=I+[\boldsymbol{w}]_{\times}$, where $[\boldsymbol{w}]_{\times} \in s o(3)$ is the skewsymmetric matrix generating the cross product by $\boldsymbol{w}=\left(w_{1}, w_{2}, w_{3}\right)^{\top}$. After 
replacing this first order approximation into the expressions for the diffusion tensors $D_{1}$ and $D_{2}$, we obtain

$$
\begin{aligned}
D_{2}(R \boldsymbol{x}+\boldsymbol{t}) & \approx D_{2}\left(\boldsymbol{x}+[\boldsymbol{w}]_{\times} \boldsymbol{x}+\boldsymbol{t}\right) \\
& \approx D_{2}(\boldsymbol{x})+D_{x}\left(\boldsymbol{e}_{1}^{\top}[\boldsymbol{w}]_{\times} \boldsymbol{x}+d_{1}\right)+D_{y}\left(\boldsymbol{e}_{2}^{\top}[\boldsymbol{w}]_{\times} \boldsymbol{x}+d_{2}\right)+D_{z}\left(\boldsymbol{e}_{3}^{\top}[\boldsymbol{w}]_{\times} \boldsymbol{x}+d_{3}\right) \\
& =D_{2}(\boldsymbol{x})+D_{x}\left(z w_{2}-y w_{3}+d_{1}\right)+D_{y}\left(x w_{3}-z w_{1}+d_{2}\right)+D_{z}\left(y w_{1}-x w_{2}+d_{3}\right) \\
R D_{1}(\boldsymbol{x}) R^{\top} & \approx\left(I+[\boldsymbol{w}]_{\times}\right) D_{1}(\boldsymbol{x})\left(I+[\boldsymbol{w}]_{\times}^{\top}\right) \approx D_{1}(\boldsymbol{x})+[\boldsymbol{w}]_{\times} D_{1}(\boldsymbol{x})+D_{1}(\boldsymbol{x})[\boldsymbol{w}]_{\times}^{\top},
\end{aligned}
$$

where $\boldsymbol{e}_{1}=(1,0,0)^{\top}, \boldsymbol{e}_{2}=(0,1,0)^{\top}$ and $\boldsymbol{e}_{3}=(0,0,1)^{\top}$. Therefore, the cost function (4) can be rewritten as

$$
J=\sum_{\Omega} \operatorname{trace}\left(U_{1} w_{1}+U_{2} w_{2}+U_{3} w_{3}+U_{4} d_{1}+U_{5} d_{2}+U_{6} d_{3}+D_{t}\right)^{2}
$$

where

$$
\begin{array}{lll}
U_{1}=-M_{1}+y D_{z}-z D_{y}, & U_{2}=-M_{2}+z D_{x}-x D_{z}, & U_{3}=-M_{3}+x D_{y}-y D_{x}, \\
U_{4}=D_{x}, & U_{5}=D_{y}, & U_{6}=D_{z}, \\
D_{t}=D_{2}(\boldsymbol{x})-D_{1}(\boldsymbol{x}), & M_{j}=\left(\left[\boldsymbol{e}_{j}\right]_{\times} D_{1}+D_{1}\left[\boldsymbol{e}_{j}\right]_{\times}^{\top}\right), & j=1, \ldots, 3 .
\end{array}
$$

After taking derivatives of $J$ with respect to $\boldsymbol{u}=\left(w_{1}, w_{2}, w_{3}, d_{1}, d_{2}, d_{3}\right)^{\top}$ and setting them to zero, we obtain the following system of linear equations on $\boldsymbol{u}$

$$
G \boldsymbol{u}=-\boldsymbol{b},
$$

where $G_{i j}=\sum_{\Omega} \operatorname{trace}\left(U_{i} U_{j}\right)$ for $i, j=1, \ldots, 6$ and $\boldsymbol{b}_{i}=\sum_{\Omega} \operatorname{trace}\left(U_{i} D_{t}\right)$ for $i=1, \ldots, 6$.

Notice that, with generic data, each voxel gives 6 linearly independent equations in 6 unknowns in $\boldsymbol{u}$. This implies that $\operatorname{rank}(G)=6$, even if $G$ is computed from a single voxel. Therefore, the minimum number of voxels needed to solve the registration problem is one, as in the translational case.

\subsection{3-D Affine Model}

Consider now the DTCC for the full affine deformation model

$$
D_{2}(A \boldsymbol{x}+\boldsymbol{t})=R D_{1}(\boldsymbol{x}) R^{\top}
$$

where $A=R S$ with $R \in S O(3)$ and $S \in \operatorname{SPSD}(3)$. If we approximate $R$ and $S$ up to first order as $R \approx I+[\boldsymbol{w}]_{\times}$and $S \approx I+\hat{s}$, where $\hat{s}$ is a symmetric matrix, we obtain the following first order approximation for $A \approx I+[\boldsymbol{w}]_{\times}+\hat{s}$. This gives

$$
\begin{aligned}
D_{2}(A \boldsymbol{x}+\boldsymbol{t}) \approx D_{2}(\boldsymbol{x}) & +D_{x}\left(\boldsymbol{e}_{1}^{\top}[\boldsymbol{w}]_{\times} \boldsymbol{x}+\boldsymbol{e}_{1}^{\top} \hat{s} \boldsymbol{x}+d_{1}\right) \\
& +D_{y}\left(\boldsymbol{e}_{2}^{\top}[\boldsymbol{w}]_{\times} \boldsymbol{x}+\boldsymbol{e}_{2}^{\top} \hat{s} \boldsymbol{x}+d_{2}\right)+D_{z}\left(\boldsymbol{e}_{3}^{\top}[\boldsymbol{w}]_{\times} \boldsymbol{x}+\boldsymbol{e}_{3}^{\top} \hat{s} \boldsymbol{x}+d_{3}\right) .
\end{aligned}
$$


Note that the only differences between this expression and the corresponding one for the rigid model are the terms involving $\hat{s}$, which can be expressed as $s_{11} x D_{x}+s_{12}\left(y D_{x}+x D_{y}\right)+s_{13}\left(x D_{z}+z D_{x}\right)+s_{22} y D_{y}+s_{23}\left(z D_{y}+y D_{z}\right)+s_{33} z D_{z}$.

Therefore, we may rewrite the cost function (44) as

$$
\begin{array}{r}
J=\sum_{\Omega} \operatorname{trace}\left(U_{1} w_{1}+U_{2} w_{2}+U_{3} w_{3}+U_{4} d_{1}+U_{5} d_{2}+U_{6} d_{3}+D_{t}+\right. \\
\left.U_{7} s_{11}+U_{8} s_{12}+U_{9} s_{13}+U_{10} s_{22}+U_{11} s_{23}+U_{12} s_{33}\right)^{2},
\end{array}
$$

where $U_{1}, \ldots, U_{6}$ are defined as in the rigid case and

$$
\begin{aligned}
& U_{7}=x D_{x}, \quad U_{8}=y D_{x}+x D_{y}, \quad U_{9}=x D_{z}+z D_{x}, \\
& U_{10}=y D_{y}, \quad U_{11}=z D_{y}+y D_{z}, \quad U_{12}=z D_{z} .
\end{aligned}
$$

After differentiating the cost function $J$ with respect to the unknown model parameters $\boldsymbol{u}=\left(w_{1}, w_{2}, w_{3}, d_{1}, d_{2}, d_{3}, s_{11}, s_{12}, s_{13}, s_{22}, s_{23}, s_{33}\right)^{\top}$ and setting the result to zero, we obtain the following linear system on $\boldsymbol{u}$

$$
G \boldsymbol{u}=-\boldsymbol{b},
$$

with $G_{i j}=\sum_{\Omega} \operatorname{trace}\left(U_{i} U_{j}\right)$ for $i, j=1, \ldots, 12$ and $\boldsymbol{b}_{i}=\sum_{\Omega} \operatorname{trace}\left(U_{i} D_{t}\right)$ for $i=1, \ldots, 12$.

Notice that, with generic data, each voxel provides 6 linearly independent equations in 12 unknowns in $\boldsymbol{u}$. This implies that $\operatorname{rank}(G)=6$ when $G$ is computed from a single voxel. Therefore, the minimum number of voxels needed to solve the registration problem is two.

\subsection{Multiscale Iterative Refinement}

The algebraic registration method presented in the previous subsections assumes implicitly that the spatial-temporal discretization of the DT image is adequate for representing the deformation of the DT volume. When this is not the case, an approach that combines motion estimates across multiple scales is needed. The existing literature on estimation of optical flow from 2-D images provides various multiscale methods for motion estimation [18. In our implementation, we adapt such methods to the case of DT data.

Our multiscale algorithm proceeds as follows:

1. Downsample $D_{1}$ and $D_{2}$ by a factor of $2^{n}$ and compute transformation $\boldsymbol{u}_{n}$ between the downsampled images by solving the linear system $G_{n} \boldsymbol{u}_{n}=-\boldsymbol{b}_{n}$.

2. Warp current $D_{1}$ to $D_{1}(\boldsymbol{x}) \leftarrow R_{n} D_{1}\left(R_{n} S_{n} \boldsymbol{x}+2^{n} \boldsymbol{t}_{n}\right) R_{n}^{\top}$, where $\left(R_{n}, S_{n}, \boldsymbol{t}_{n}\right)$ are the deformation parameters corresponding to $\boldsymbol{u}_{n}$.

3. If $n \geq 1$, set $n \leftarrow n-1$ and go to 1 .

4. Set $\bar{u}=\sum_{i=0}^{n}\left(w_{1}, w_{2}, w_{3}, 2^{i} d_{1}, 2^{i} d_{2}, 2^{i} d_{3}, s_{11}, s_{12}, s_{13}, s_{22}, s_{23}, s_{33}\right)_{i}^{\top}$, where $\boldsymbol{u}_{i}=\left(w_{1}, w_{2}, w_{3}, d_{1}, d_{2}, d_{3}, s_{11}, s_{12}, s_{13}, s_{22}, s_{23}, s_{33}\right)_{i}^{\top}$.

Notice that in transforming the motion parameters from one scale to another only the translational part of the affine deformation is affected. This is because when scaling the voxel coordinates by a factor $\lambda$ we obtain $\lambda \boldsymbol{x}_{2}=A \lambda \boldsymbol{x}_{1}+\lambda \boldsymbol{t}$, thus $\boldsymbol{t}$ is scaled, but $A$ is not. 


\section{Feature-Based Registration of Diffusion Tensor Images}

Consider now the registration of diffusion tensor images from a set of point correspondences in two images $\boldsymbol{x}_{1} \leftrightarrow \boldsymbol{x}_{2}$. If we are given 4 or more point correspondences, then recovering a global deformation model $(A, \boldsymbol{t})$ from the equation $\boldsymbol{x}_{2}=A \boldsymbol{x}_{1}+\boldsymbol{t}$ is simply a linear problem. However, this linear solution does not make use of any tensor information, because the constraints due to tensor reorientation are not incorporated. Since a point correspondence $\boldsymbol{x}_{1} \leftrightarrow \boldsymbol{x}_{2}$ naturally induces a tensor correspondence $D_{1} \leftrightarrow D_{2}$, we propose a feature-based registration method that exploits both point and tensor correspondences. In fact, we show that in spite of the need for a tensor reorientation model, the registration problem can still be solved linearly.

\subsection{Rigid Registration from Point and Tensor Correspondences}

Under a rigid transformation $(R, \boldsymbol{t}) \in S E(3)$, where $R \in S O(3)$ is the rotation and $\boldsymbol{t} \in \mathbb{R}^{3}$ is the translation, the two images are related by the equations

$$
\boldsymbol{x}_{2}=R \boldsymbol{x}_{1}+\boldsymbol{t} \quad \text { and } \quad D_{2}=R D_{1} R^{\top} .
$$

From the first equation in (15), note that if $R$ were known, we could immediately solve for $\boldsymbol{t}$ as $\boldsymbol{x}_{2}-R \boldsymbol{x}_{1}$. Thus, solving the registration problem for a single voxel boils down to estimating the rotation matrix from the second equation in (15).

To this end, consider the SVD of the diffusion tensors $D_{1}=U_{1} \Sigma_{1} U_{1}^{\top}$ and $D_{2}=U_{2} \Sigma_{2} U_{2}^{\top}$. In the absence of noise, the equation $D_{2}=R D_{1} R^{\top}$ implies that $D_{1}$ and $D_{2}$ share the same singular values, i.e. $\Sigma_{1}=\Sigma_{2}$. In addition, we have

$$
U_{2} \Sigma_{2} U_{2}^{\top}=R U_{1} \Sigma_{1} U_{1}^{\top} R^{\top} \Longrightarrow \Sigma_{2}=U_{2}^{\top} R U_{1} \Sigma_{1} U_{1}^{\top} R^{\top} U_{2} .
$$

Therefore, if the three singular values of $D_{1}$ are different, we can immediately solve for the rotation as $R=U_{2} U_{1}^{\top}$ or $R=-U_{2} U_{1}^{\top}$, depending on whether $\operatorname{det}\left(U_{2} U_{1}^{\top}\right)=1$ or not.

In the presence of noise, the matrices $\Sigma_{1}$ and $\Sigma_{2}$ will not necessarily coincide, thus we seek a rotation $R$ that minimizes the error in (4), which in the case of a single correspondence is given by $\operatorname{trace}\left(D_{2}-R D_{1} R^{\top}\right)^{2}$. Minimizing this error is equivalent to solving the following optimization problem

$$
\max _{R} \operatorname{trace}\left(D_{2} R D_{1} R^{\top}\right)=\max _{R} \operatorname{trace}\left(U_{2} \Sigma_{2} U_{2}^{\top} R U_{1} \Sigma_{1} U_{1}^{\top} R^{\top}\right) .
$$

We now prove that the solution to this optimization problem is $R=U_{2} U_{1}^{\top}$ or $R=-U_{2} U_{1}^{\top}$ as well. The proof will follow from the following theorem [19].

Theorem 1. Let $A, B \in \mathbb{R}^{n \times n}$. If both $A B$ and $B A$ are positive semidefinite, then there exists a permutation $\tau$ of the integers $1,2, \ldots, n$, such that

$$
\operatorname{trace}(A B)=\sum_{i=1}^{n} \sigma_{i}(A) \sigma_{\tau(i)}(B) \leq \sum_{i=1}^{n} \sigma_{i}(A) \sigma_{i}(B),
$$

where $\left\{\sigma_{i}(C)\right\}_{i=1}^{n}$ are the singular values of $C$ arranged in non-decreasing order. 
By applying Theorem 1 to equation (16) with $A=D_{2}$ and $B=R D_{1} R^{\top}$, we obtain $\operatorname{trace}\left(D_{2} R D_{1} R^{\top}\right)=\sum_{i=1}^{n} \sigma_{i}\left(D_{1}\right) \sigma_{\tau(i)}\left(D_{2}\right)=\sum_{i=1}^{n} \sigma_{i}\left(\Sigma_{1}\right) \sigma_{\tau(i)}\left(\Sigma_{2}\right) \leq \operatorname{trace}\left(\Sigma_{1} \Sigma_{2}\right)$.

If we now substitute $R= \pm U_{2} U_{1}^{\top}$ on the left hand side we get

$$
\begin{aligned}
\operatorname{trace}\left(D_{2} R D_{1} R^{\top}\right) & =\operatorname{trace}\left(U_{2} \Sigma_{2} U_{2}^{\top} U_{2} U_{1}^{\top} U_{1} \Sigma_{1} U_{1}^{\top} U_{1} U_{2}^{\top}\right) \\
& =\operatorname{trace}\left(U_{2} \Sigma_{2} \Sigma_{1} U_{2}^{\top}\right)=\operatorname{trace}\left(\Sigma_{2} \Sigma_{1}\right) .
\end{aligned}
$$

Hence the rotation matrix $R=U_{2} U_{1}^{\top}$ or $R=-U_{2} U_{1}^{\top}$ achieves the maximum, as claimed. Notice that in order for the maximum to be unique, it is necessary that both $D_{1}$ and $D_{2}$ have different singular values, so that $U_{1}$ and $U_{2}$, hence $R$, are uniquely defined.

In the case of $N$ correspondences, we seek to find the optimal rotation that minimizes $\sum_{i=1}^{N} \operatorname{trace}\left(D_{2 i}-R D_{1 i} R^{\top}\right)^{2}$. We are not aware of an exact solution to this problem. We compute an approximate solution by averaging the rotations $R_{i}=U_{2 i} U_{1 i}^{\top}$ computed from the individual correspondences. We use the method in [20] for computing the average rotation.

\subsection{Affine Registration from Point and Tensor Correspondences}

We now extend the registration method from a rigid to an affine deformation

$$
\boldsymbol{x}_{2}=A \boldsymbol{x}_{1}+\boldsymbol{t} \quad \text { and } \quad D_{2}=R D_{1} R^{\top} .
$$

First, we proceed as in the rigid case in order to obtain the rotation matrix $R$ from the DTs. Since $A$ can be expressed uniquely as $A=R S$, where $R \in$ $S O(3)$ and $S \in \operatorname{SPSD}(3)$, there are 6 independent parameters in $S$ and 3 in $\boldsymbol{t}$. Since each point correspondence $\left(\boldsymbol{x}_{1}, \boldsymbol{x}_{2}\right)$ provides 3 equations, we will need 3 correspondences in order to solve for $S$ and $\boldsymbol{t}$. More specifically, let $\left(\boldsymbol{x}_{11}, \boldsymbol{x}_{21}\right)$, $\left(\boldsymbol{x}_{12}, \boldsymbol{x}_{22}\right)$ and $\left(\boldsymbol{x}_{13}, \boldsymbol{x}_{23}\right)$ be such correspondences. We can solve for $S$ and $\boldsymbol{t}$ linearly from

$$
\boldsymbol{x}_{21}=R S \boldsymbol{x}_{11}+\boldsymbol{t}, \quad \boldsymbol{x}_{22}=R S \boldsymbol{x}_{12}+\boldsymbol{t}, \quad \text { and } \quad \boldsymbol{x}_{23}=R S \boldsymbol{x}_{13}+\boldsymbol{t} .
$$

\section{Experimental Results}

We evaluate the performance of the proposed registration algorithm on a real DT image of the human brain 21. The image size is $148 \times 190 \times 160$ voxels and the voxel size is $1 \mathrm{~mm} \times 1 \mathrm{~mm} \times 1 \mathrm{~mm}$. The implementation is done in a hierarchy where we start downsampling at scale of $2^{2}$. At this scale, a window $\Omega$ of size $5 \times 5 \times 5$ is used for the local computation of the deformation. For the subsequent scale of $2^{1}$, the window size is $11 \times 11 \times 11$, followed by $21 \times 21 \times 21$ at the original resolution. Fig. 1 shows the estimated transformation at each 


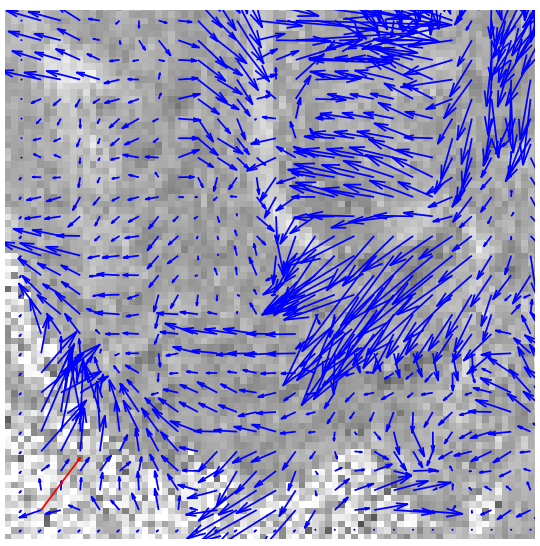

(a) Deformation at scale $2^{2}$

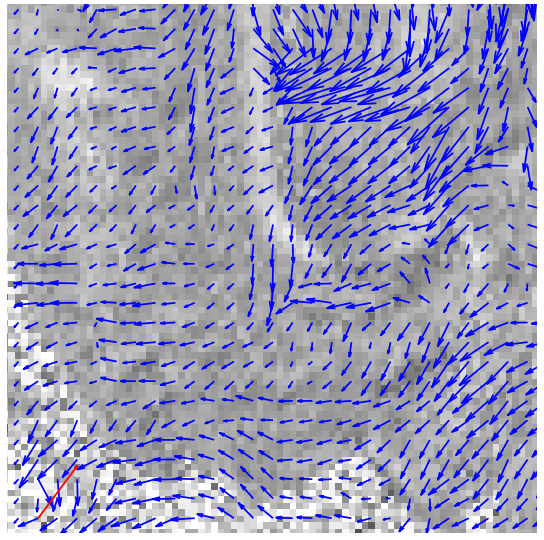

(b) Deformation at scale $2^{1}$

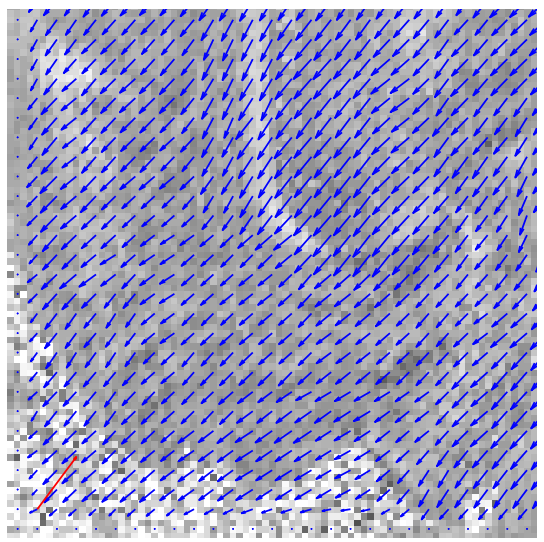

(c) Final deformation

Fig. 1. Zoomed-in results for a DT brain image

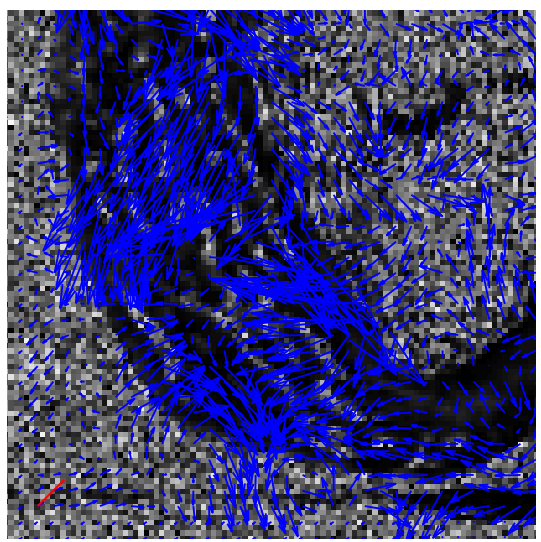

(a) Deformation at scale $2^{2}$

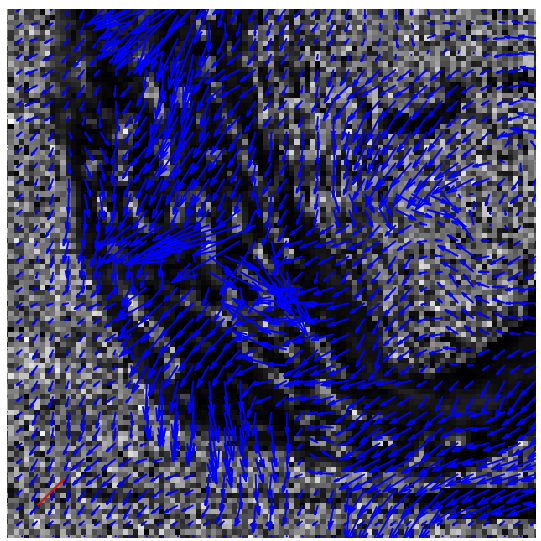

(b) Deformation at scale $2^{1}$

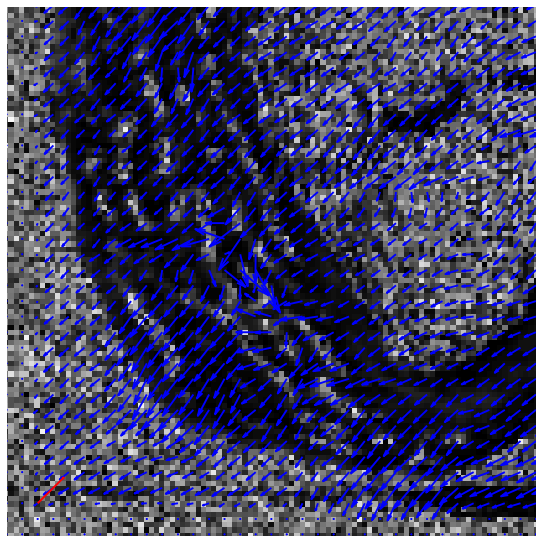

(c) Final deformation

Fig. 2. Zoomed-in results of a DT heart image 
scale for a translational deformation of $\boldsymbol{t}=(0,8,8)$ voxels. It can be seen that the DTCC-based method correctly estimates the direction of the deformation for most of the image, though it usually underestimates the magnitude. The method performs significantly poorer in regions where there is a sharp variation of the tensor field along a 1-D curve or a 2-D surface, e.g., in boundary regions, or regions where the tensor data has low variability. This is because the $G$ matrix, from which the deformation field is estimated, is rank deficient in these regions. This is simply a generalization of the well-known aperture problem in standard 2-D images, which refers to the impossibility of estimating optical flow in regions with low texture using a local method.

Similar experiments are done on a DT image of a human heart obtained from 22. Fig. 2 shows the estimated deformation field for a translational deformation of $\boldsymbol{t}=(0,5,5)$. Notice that the performance of the algorithm on the heart dataset is worse than in the brain dataset. This is expected as the proportion of

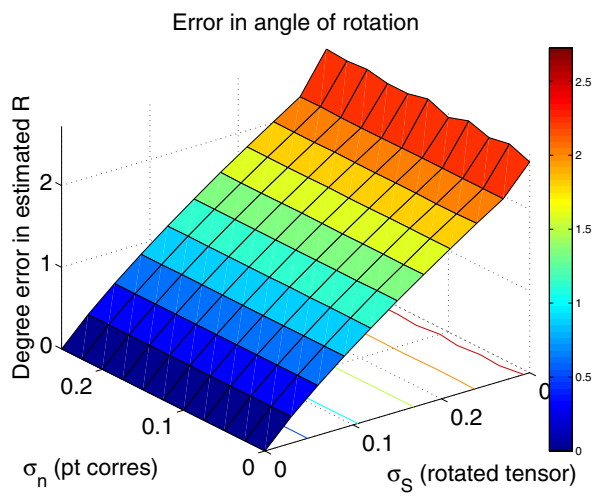

(a) $\mathrm{R}$ error (degrees) vs noise

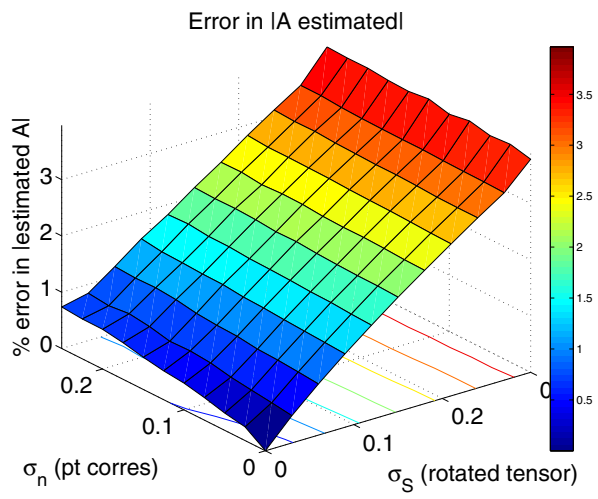

(c) $\%$ error in A vs noise

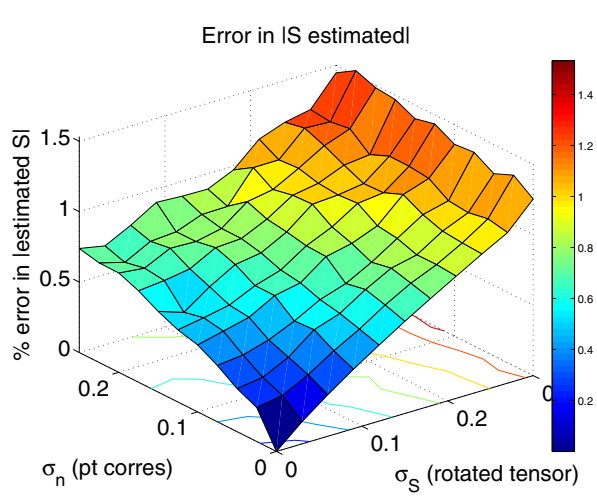

(b) $\%$ error in $\mathrm{S}$ vs noise

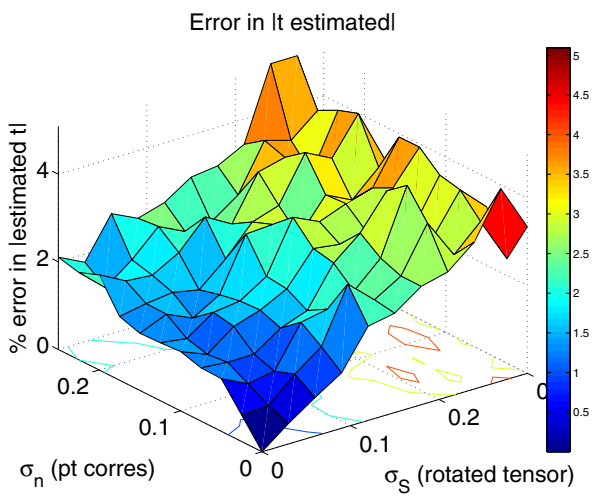

(d) $\%$ error in $\boldsymbol{t}$ vs noise

Fig. 3. Error in estimated $R, S, A$ and $\boldsymbol{t}$ vs $\sigma_{n}$ and $\sigma_{s}$ 
the volume of the heart data that contains high variability and is away from the boundaries is significantly smaller.

Finally, we evaluate the proposed feature-based affine registration algorithm on synthetic data for varying levels of noise on the image data. We generate a $20 \times 20 \times 20$ volume containing the tensor $R_{\text {rand }} \operatorname{diag}\left\{[10 ; 5 ; 2]+\boldsymbol{t}_{\text {rand }}\right\} R_{\text {rand }}^{\top}$, where $R_{\text {rand }}$ is a rotation matrix with an arbitrary rotation at each voxel and $\boldsymbol{t}_{\text {rand }} \in \mathbb{R}^{3}$ is a translation vector whose entries are distributed uniformly in $[0,1]$. Given an affine transformation $(A, \boldsymbol{t})$ with $A=R S$, we construct the second volume by applying the transformation $\boldsymbol{x}_{2}=A \boldsymbol{x}_{1}+\boldsymbol{t}+\boldsymbol{n}$ to the voxel coordinates, where $\boldsymbol{n} \sim N\left(\mathbf{0}, \sigma_{n}^{2} I\right)$, and the transformation $D_{2}=R U_{1}\left(\Sigma_{1}+S_{n}\right) U_{1}^{\top} R^{\top}$ to the tensor data, where $D_{1}=U_{1} \Sigma_{1} U_{1}^{\top}$ is the SVD of $D_{1}$ and $S_{n}=\sigma_{s} R_{\text {small }} \Sigma_{1} R_{\text {small }}^{\top}$. $\sigma_{s}$ is the amount of noise, and $R_{\text {small }}$ is a rotation matrix generated via the exponential map by $R_{\text {small }}=\exp \left(\sigma_{s}\left|\boldsymbol{w}_{1}\right| \boldsymbol{v}\right)$, where $\boldsymbol{v} \in \mathbb{R}^{3}$ is a unit random vector and $\left[\boldsymbol{w}_{1}\right]_{\times}=\log U_{1}$.

We apply our algorithm to the so-generated data for varying the levels of noise $\sigma_{n}$ and $\sigma_{s}$. For each noise level, we calculated the percentage of error as $|p-\hat{p}| /|\hat{p}| \times 100$, where $p$ is $A, S$ or $\boldsymbol{t}$ and $\hat{p}$ is the corresponding true parameter. The rotation error is calculate as $\cos ^{-1}\left(\left(\operatorname{trace}\left(R \hat{R}^{\top}\right)-1\right) / 2\right)$. Fig. 3 shows the mean errors over 30 trials. As expected, the errors increase as a function of noise. Also, note that when $\sigma_{s}=0$, the rotation error is zero for all $\sigma_{n}$, as expected.

\section{$5 \quad$ Summary and Conclusions}

We have presented a closed form solution to the registration of diffusion tensor images. The first contribution of this paper is to show that by using the diffusion tensor constancy constraint (DTCC), it is possible to have a linear relationship between the deformation parameters, the tensor data and its first order derivatives. Comparing our results on brain and heart DT images to those in 23], it is apparent that the multiscale algebraic approach presented in this paper is able to better estimate the deformation parameters. However, there is still much work to be done in order to improve the accuracy of the estimated transformation.

The second contribution of this paper is to show that if point correspondences are known, then it is again easy to find the deformation parameters $A, R, \boldsymbol{t}$ by solving a set of linear equations. The computational complexity of both linear methods is significantly smaller compared to gradient descent methods.

\section{Acknowledgments}

This work was partially supported by Hopkins WSE startup funds and by grant NSF CAREER IIS-0447739. 


\section{References}

1. Mori, S., van Zijl, P.: Fiber tracking: principles and strategies - a technical review. NMR in Biomedicine 15 (2002) 468-480

2. Lazar, M., et al. : White matter tractography using diffusion tensor deflection. In: Human Brain Mapping 18:306-321. (2003)

3. Vemuri, B., et al. : Fiber tract mapping from diffusion tensor MRI. In: IEEE workshop on Variational and Level Set Methods in Computer Vision. (2001)

4. Lori, N.F., et al. : Diffusion tensor fiber tracking of human brain connectivity: aquisition methods, reliability analysis and biological results. NMR in Biomedicine 15 (2002) 494-515

5. Lal, R.: Probabilistic cortical and myocardinal fiber tracking in diffusion tensor imaging. Master's thesis, The Johns Hopkins University (2001)

6. Wang, Z., Vemuri, B.: An affine invariant tensor dissimilarity measure and its applications to tensor-valued image segmentation. IEEE Conference on Computer Vision and Pattern Recognition (2004)

7. Zhang, H., Yushkevich, P., Gee, J.: Registration of diffusion tensor images. IEEE Conference on Computer Vision and Pattern Recognition (2004)

8. Ding, Z., et al. : Classification and quantification of neuronal fiber pathways using diffusion tensor MRI. Magnetic Resonance in Medicine 49 (2003) 716-721

9. Brun, A., Knutsson, H., Park, H.J., Shenton, M.E., Westin, C.F.: Clustering fiber tracts using normalized cuts. In: MICCAI. (2004) 368-375

10. Shi, J., Malik, J.: Normalized cuts and image segmentation. IEEE Trans. on Pattern Analysis and Machine Intelligence 22 (2000)

11. Zhukov, L., Museth, K., Breen, D., Whitaker, R., Barr, A.: Level set segmentation and modeling of DT-MRI human brain data. Journal of Electronic Imaging (2003)

12. Alexander, D., et al. : Spatial transformation of diffusion tensor magnetic resonance images. IEEE Transactions on Medical Imaging 20 (2001) 1131-1139

13. Ruiz-Alzola, J., et al. : Nonrigid registration of 3D tensor medical data. Medical Image Analysis 6 (2002) 143-161

14. Guimond, A., et al. : Deformable registration of DT-MRI data based on transformation invariant tensor characteristics. In: IEEE International Symposium on Biomedical Imaging. (2002)

15. Park, H.J., et al. : Spatial normalization of diffusion tensor MRI using multiple channels. Neuroimage 20 (2003) 1995-2009

16. Gallier, J.: Geometric Methods and Applications for Computer Science and Engineering. Springer-Verlag New York (2001)

17. Murray, R.M., Li, Z., Sastry, S.S.: A Mathematical Introduction to Robotic Manipulation. CRC Press Inc. (1994)

18. Battiti, R., Amaldi, E., Koch, C.: Computing optical flow across multiple scales: An adaptive coarse-to-fine strategy. International Journal of Computer Vision 6 (1991) 133-145

19. Horn, R., Johnson, C.: Matrix Analysis. Cambridge University Press (1985)

20. Fletcher, P.T., et al. : Principal geodesic analysis for the study of nonlinear statistics of shape. IEEE Transactions on Medical Imaging 23 (2004) 995-1005

21. Kindlmann, G., Alexander, A.: Brain dataset, University of Utah, and University of Wisconsin-Madison. (http://www.sci.utah.edu/ gk/DTI-data/)

22. Helm, P.A., Winslow, R.L., McVeigh, E.: Cardiovascular DTMRI data sets. (http://www.ccbm.jhu.edu)

23. Goh, A., Vidal, R.: An algebraic solution to rigid registration of diffusion tensor images. In: IEEE International Symposium on Biomedical Imaging. (2006) 